\title{
isomiRs: Increasing Evidences of isomiRs Complexity in Plant Stress Functional Biology
}

\author{
Gaurav Sablok $^{1 *}$, Ashish K. Srivastva ${ }^{2}$, Penna Suprasanna ${ }^{2}$, Vesselin Baev ${ }^{3}$ and \\ Peter J. Ralph ${ }^{1}$ \\ ${ }^{1}$ Plant Functional Biology and Climate Change Cluster (C3), University of Technology Sydney, Sydney, NSW, Australia, \\ ${ }^{2}$ Nuclear Agriculture and Biotechnology Division, Bhabha Atomic Research Centre, Mumbai, India, ${ }^{3}$ Department of Plant \\ Physiology and Molecular Biology, University of Plovdiv, Plovdiv, Bulgaria
}

Keywords: biotic and abiotic stress, development, miRNAs, plants, isomiRs, smallRNA-seq

\section{miRNAs AND isomiRs: RISE OF COMPLEX miRNA ISOFORMS}

How and by which mechanism plants control post-transcriptional regulation? Reinhart et al. (2002) pinpointed the role of non-coding, small endogenous regulatory microRNAs (miRNAs) as the regulatory switch that controls the post-transcriptional regulation. Since then, several families of regulatory miRNAs including artificial miRNAs (Sablok et al., 2011) have been discovered and have shown to play a key role as system wide regulators, which are activated in response to several biotic and abiotic stress adaptations (Zhang, 2015), as well as regulators of plant development (Reinhart et al., 2002; Sun, 2012). Several reports on the identification and characterization of miRNAs have been published so far (for a review, see Budak et al., 2015), which established miRNAs as major post-transcriptional regulatory factors in plants system biology. Endogenous miRNAs perform regulatory role by binding specifically to the 3'-UTR of mRNAs, which in turns triggers the degradation of the targeted transcript, thereby leading to the shutdown of protein translation. Endogenous miRNAs originate from the stem of a single-stranded stem-loop precursor as a miRNA/miRNA* duplex with an approximately 2 -nt $3^{\prime}$-end overhang. However, not all small RNAs expressed from a stem-loop are necessarily either the precise miRNA or precise miRNA*.

The advent of the high throughput small RNA sequencing approaches and concurrent development in the identification of miRNAs using advanced algorithmic-based computational approaches has led to the discovery of a new class of regulatory RNAs called isomiRs, which are canonical variants of miRNAs (Morin et al., 2008b). The biogenesis of these canonical miRNA sequence variants in plants might be due to the imprecise cleavage activity by the Rnase III enzyme or due to the post-transcriptional RNA editing events (Hackenberg et al., 2013) or by nucleotidyl transferases (Wyman et al., 2011). Morin et al. (2008a) hypothesized the origin of isomiRs as cleavage site variability in the pre-miRNA hairpin, which is cleaved by either DICER1 or DROSHA (Morin et al., 2008b).

Taking into account the length and nucleotide variation along with the non-templated additions (NTAs) such as adenylation and uridylation at the $3^{\prime}$-end with non-random functionality (Wyman et al., 2011), isomiRs can be classified into $5^{\prime}$ isomiR, $3^{\prime}$ isomiR, and polymorphic isomiRs (Neilsen et al., 2012; Jeong et al., 2013). Global analysis of the isomiRs and canonical miRNAs has shown uridine to be the preferential nucleotide at $5^{\prime}$ - and $3^{\prime}$-ends. This has led to the conclusion that isomiRs commonly feature " $\mathrm{U}-\mathrm{C}$ " at the $3^{\prime}$-end of the isomiRs as opposed to the addition of $\mathrm{C}$ at the $3^{\prime}$-end of the plant miRNAs (Zhang et al., 2013). However, isomiRs displayed a frequent truncation of the cytodine from both the ends, presenting a new complex cytodine balance in isomiRs (Xie et al., 2015), which suggests that uridylation plays a role in avoiding degradation. The primary sequences of identified isomiRs so far differ from the indexed high content miRBase miRNAs 
in their $5^{\prime}$-end (changing the "seed" region and suggesting a different target molecule) or in their $3^{\prime}$-end or both. The question that why this variant occurs is still intriguing and challenging, and needs to be addressed. However, recent reports have started suggesting the target cleavage efficiency of isomiRs, thus establishing them as another class of regulatory functional RNAs.

\section{isomiRs IDENTIFICATION AND INTER-PLAY IN PLANT STRESS FUNCTIONAL GENOMICS}

On the basis of accumulated knowledge about isomiRs, as well as their abundance and involvement in target cleavage (Ahmed et al., 2014), several algorithms either as web-based or standalone tool have been developed, using read mapping approaches to delineate the repertoire of isomiRs abundance and expression (Table 1). Previously, we developed the first web-based tool, isomiRex (bioinfol.uni-plovdiv.bg/isomiRex/), which provides the high-throughput classification and differential expression of isomiRs and supports a broad range of organisms including plants (Sablok et al., 2013). Following isomiRex, several tools have been developed such as IsomiRage (Muller et al., 2014) and isomiRID (de Oliveira et al., 2013), which can distinguish isomiRs using template-based and non-template-based predictions. Since isomiRs can be a result of the adenylation or uridylation events, IsomiRage (Muller et al., 2014) implements algorithmic identification and classification of functionally relevant isomiRs based on their adenylation, uridylation, and other respective biological events (Muller et al., 2014).

Whether isomiRs are functional or just variants has been widely discussed and debated since the first reported evidence of isomiRs in Oryza sativa (Morin et al., 2008b). Although their functional role and capabilities are still unknown, isomiRs have the potential to extend the canonical miRNA regulatory network. This adds credence to the hypothesis that "mature miRNAs" cannot be related to only a single individual sequence and that a single precursor may cleave more than one functional product. Profiling of tissue-specific small RNAs in Peach (Prunus

TABLE 1 | Recently developed classification tools for identifying isomiRs.

\begin{tabular}{|c|c|c|c|}
\hline Tool & Address & Type & References \\
\hline SeqCluster & $\begin{array}{l}\text { https://github.com/ } \\
\text { Ipantano/seqbuster }\end{array}$ & Standalone & Pantano et al., 2011 \\
\hline miRSeqNovel & $\begin{array}{l}\text { http://sourceforge.net/ } \\
\text { projects/mirseq/files }\end{array}$ & Standalone & Qian et al., 2012 \\
\hline isomiRID & $\begin{array}{l}\text { http://www.ufrgs.br/ } \\
\text { RNAi/isomiRID/ }\end{array}$ & Standalone & de Oliveira et al., 2013 \\
\hline isomiRex & $\begin{array}{l}\text { bioinfo1.uni-plovdiv. } \\
\text { bg/isomiRex/ }\end{array}$ & Web-based & Sablok et al., 2013 \\
\hline miRspring & $\begin{array}{l}\text { http://mirspring. } \\
\text { victorchang.edu.au }\end{array}$ & Standalone & Humphreys and Suter, 2013 \\
\hline IsomiRage & $\begin{array}{l}\text { http://cru.genomics.iit. } \\
\text { it//somirage/ }\end{array}$ & Standalone & Muller et al., 2014 \\
\hline sRNAtoolbox & $\begin{array}{l}\text { http://bioinfo5.ugr.es/ } \\
\text { srnatoolbox }\end{array}$ & Standalone & Rueda et al., 2015 \\
\hline
\end{tabular}

Persica L.) revealed an abundance of tissue-specific isomiRs (392 isomiRs-miRNA and miRNA*-related-corresponding to 26 putative miRNA coding loci), which supports the hypothesis that the origin of isomiRs is not just a random functional event in plant post-transcriptional machinery (Colaiacovo et al., 2012). Realizing the potential of isomiRs and their ability to cleave targets (Ahmed et al., 2014), several studies started exploring their functional role in plants. In Phaseolus vulgaris, as many as 57 functional isomiRs spanning across 25 families have been identified in nodule development and phasiRNAs generation (Formey et al., 2015).

Alongside the discovery of isomiRs and their ability to cleave targets, studies pertaining to the relative differential expression of isomiRs have also been conducted. Jeong et al. (2013) reported strong expression of isomiRs with $5^{\prime}$ variations in $A$. thaliana using Parallel Analysis of RNA Ends (PARE-Seq), co-immunoprecipitation, and ARGONAUTE (AGO) loading data (Jeong et al., 2013). Using a reversed framework approach and degradome analysis, Shao et al. (2015) revealed the higher expression of the isomiRs as compared to their canonical miRNAs in Oryza sativa (Shao et al., 2015). Interestingly, they showed high abundance of iso-osa-miR528$5 \mathrm{p}$ in AGO1 complexes as compared to the osa-miR528-5p (1315 rpm vs. $165.72 \mathrm{rpm}$ ) (Shao et al., 2015). Ehya et al. (2013) observed differential expression of several isomiRs of canonical miRNAs involved in auxin signaling, such as miR160 (for miR160*), miR166, and miR167 suggesting miRNA-mediated auxin signaling, which might regulate the response of the Mexican Lime tree to Phytoplasma (Ehya et al., 2013). Taking these studies into account, it can be concluded that isomiRs are not random events and might play a key role in increasing the miRNAome complexity in plants.

In addition to the developmental and tissue-specific functions (Colaiacovo et al., 2012), isomiRs play an important role in modulating and regulating the miRNAome in biotic and abiotic stress conditions. However, as compared with the widely demonstrated regulatory roles of the differentially expressed, conserved and novel miRNAs in biotic and abiotic stress conditions in plants (Zhang, 2015), limited reports have shown the differential expression of the isomiRs (Baev et al., 2014). Nonetheless, it is worthwhile to mention that recent reports in stress conditions have presented the evidences of isomiRs expression along with the expression of the canonical miRNAs in model plants. The information gleaned from the recent phosphorus (P) deficiency stress studies in Hordeum vulgare (Barley) have highlighted the up-regulated isomiRs of miR399 and miR827 family under P deficiency significantly (Hackenberg et al., 2013). Understanding the post-transcriptional regulation of the signaling pathways during temperature stress response is critical in dealing global climate change. Baev et al. (2014) recently demonstrated the differential regulation of the miR160c isomiRs in high- and low-temperature conditions in Arabidopsis thaliana with the identified isomiRs showing substantially higher expression as compared to the canonical miRNAs. Such regulated isomiRs under P deficiency and high- and low-temperature stress suggest that isomiRs play a role in regulating the miRNAome in stress-induced biological gene regulation. 
In addition to the relative recent increase in the knowledge gain about isomiRs, their biogenesis and expression, significant efforts have been made to understand the role of the functional target cleavage capacity of the isomiRs. Since reported variation in isomiRs sequences occurs at the $3^{\prime}$ - or $5^{\prime}$-ends, they could potentially bind to a different repertoire of targets relative to their mature reference counterparts. The higher expression of the isomiRs as compared to the canonical mature miRNA may affect the target cleavage efficiency of that particular miRNA. Using PARE-Seq, Jeong et al. (2013) demonstrated the differential target cleavage capacity of miR161.1 isomiRs. In model plant $A$. thaliana, differential binding capacities of the isomiRs as compared to the canonical miRNAs, as well as high efficiency of the isomiRs in target cleavage as compared to the canonical miRNAs, have been recently demonstrated (Ahmed et al., 2014). In addition, higher target prediction efficiency using coupled combinations of miRNAs and isomiRs has been observed in A. thaliana (Ahmed et al., 2014). Although less evidence have been shown toward the functional gain or loss of the isomiRs in plants, they have been confirmed experimentally in other models systems including humans, thus representing the role of isomiRs as evolutionary and functionally important variants (Tan et al., 2014; Cammaerts

\section{REFERENCES}

Ahmed, F., Senthil-Kumar, M., Lee, S., Dai, X., Mysore, K. S., and Zhao, P. X. (2014). Comprehensive analysis of small RNA-seq data reveals that combination of miRNA with its isomiRs increase the accuracy of target prediction in Arabidopsis thaliana, RNA. Biology (Basel). 11, 1414-1429. doi: 10.1080/15476286.2014.996474

Baev, V., Milev, I., Naydenov, M., Vachev, T., Apostolova, E., Mehterov, N., et al. (2014). Insight into small RNA abundance and expression in high- and low-temperature stress response using deep sequencing in Arabidopsis. Plant Physiol. Biochem. 84, 105-114. doi: 10.1016/j.plaphy.2014.09.007

Budak, H., Kantar, M., Bulut, R., and Akpinar, B. A. (2015). Stress responsive miRNAs and isomiRs in cereals. Plant Sci. 235, 1-13. doi: 10.1016/j.plantsci.2015.02.008

Cammaerts, S., Strazisar, M., De Rijk, P., and Del Favero, J. (2015). Genetic variants in microRNA genes: impact on microRNA expression, function, and disease. Front. Genet. 6:186. doi: 10.3389/fgene.2015.00186

Colaiacovo, M., Bernardo, L., Centomani, I., Crosatti, C., Giusti, L., Orrù, L., et al. (2012). A survey of MicroRNA length variants contributing to miRNome complexity in peach (Prunus Persica, L.). Front. Plant Sci. 3:165. doi: 10.3389/fpls.2012.00165

de Oliveira, L. F., Christoff, A. P., and Margis, R. (2013). isomiRID: a framework to identify microRNA isoforms. Bioinformatics 29, 2521-2523. doi: 10.1093/bioinformatics/btt424

Ehya, F., Monavarfeshani, A., Mohseni Fard, E., Karimi Farsad, L., Khayam Nekouei, M., Mardi, M., et al. (2013). Phytoplasma-responsive microRNAs modulate hormonal, nutritional, and stress signalling pathways in mexican lime trees. PLoS ONE 8:e66372. doi: 10.1371/journal.pone. 0066372

Formey, D., Iñiguez, L. P., Peláez, P., Li, Y. F., Sunkar, R., Sánchez, F., et al. (2015). Genome-wide identification of the Phaseolus vulgaris sRNAome using small RNA and degradome sequencing. BMC Genomics. 16:423. doi: 10.1186/s12864015-1639-5

Hackenberg, M., Shi, B. J., Gustafson, P., and Langridge, P. (2013). Characterization of phosphorus-regulated miR399 and miR827 and their isomirs in barley under phosphorus-sufficient and phosphorus-deficient conditions. BMC Plant Biol. 13:214. doi: 10.1186/1471-2229-13-214 et al., 2015). In conclusion, isomiRs act as canonical partners to miRNAs in regulating developmental and stress-associated post-transcriptional responses in stress. Realizing the emerging occurrences and the read-based support for the detection of the isomiRs, it can be presumed that post-transcriptionally isomiRs and canonical miRNAs act synergistically to regulate the developmental and signaling pathways in plants.

\section{AUTHORS CONTRIBUTIONS}

GS and AS conceived the idea, GS drafted the opinion article, PS, $\mathrm{VB}$ and $\mathrm{PR}$ provided revisions to the article.

\section{ACKNOWLEDGMENTS}

GS thanks Plant Functional and Climate Change Cluster (C3) for providing the computational facilities and bioinformatics infrastructure. This opinion was supported from the Plant and Functional Climate Change Cluster Internal Start up grant number: 2226018 to GS. GS thanks handling Editor Dr. Manoj Prasad and three reviewers for providing critical suggestions for the improvement of the manuscript.

Humphreys, D. T., and Suter, C. M. (2013). miRspring: a compact standalone research tool for analyzing miRNA-seq data. Nucleic Acids Res. 41:e147. doi: 10.1093/nar/gkt485

Jeong, D. H., Thatcher, S. R., Brown, R. S., Zhai, J., Park, S., Rymarquis, L. A., et al. (2013). Comprehensive investigation of microRNAs enhanced by analysis of sequence variants, expression patterns, ARGONAUTE loading, and target cleavage. Plant Physiol. 162, 1225-1245. doi: 10.1104/pp.113.219873

Morin, R. D., Aksay, G., Dolgosheina, E., Ebhardt, H. A., Magrini, V., Mardis, E. R., et al. (2008b). Comparative analysis of the small RNA transcriptomes of Pinus contorta and Oryza sativa. Genome Res. 18, 571-584. doi: 10.1101/gr.6897308

Morin, R. D., O’Connor, M. D., Griffith, M., Kuchenbauer, F., Delaney, A., Prabhu, A. L., et al. (2008a). Application of massively parallel sequencing to microRNA profiling and discovery in human embryonic stem cells. Genome Res. 18, 610-621. doi: 10.1101/gr.7179508

Muller, H., Marzi, M. J., and Nicassio, F. (2014). IsomiRage: from functional classification to differential expression of miRNA isoforms. Front. Bioeng. Biotechnol. 2:38. doi: 10.3389/fbioe.2014.00038

Neilsen, C. T., Goodall, G. J., and Bracken, C. P. (2012). IsomiRs-the overlooked repertoire in the dynamic microRNAome. Trends Genet. 28, 544-549 doi: 10.1016/j.tig.2012.07.005

Pantano, L., Estivill, X., and Martí, E. (2011). A non-biased framework for the annotation and classification of the non-miRNA small RNA transcriptome. Bioinformatics 27, 3202-3203. doi: 10.1093/bioinformatics/ btr527

Qian, K., Auvinen, E., Greco, D., and Auvinen, P. (2012). miRSeqNovel: an R based workflow for analyzing miRNA sequencing data. Mol. Cell. Probes. 26, 208-2011. doi: 10.1016/j.mcp.2012.05.002

Reinhart, B. J., Weinstein, E. G., Rhoades, M. W., Bartel, B., and Bartel, D. P. (2002). MicroRNAs in plants. Genes Dev. 16, 1616-1626. doi: $10.1101 / \operatorname{gad} .1004402$

Rueda, A., Barturen, G., Lebrón, R., Gómez-Martín, C., Alganza, Á., Oliver, J. L., et al. (2015). sRNAtoolbox: an integrated collection of small RNA research tools. Nucleic Acids Res. 43, W467-W473. doi: 10.1093/nar/gkv555

Sablok, G., Milev, I., Minkov, G., Minkov, I., Varotto, C., Yahubyan, G., et al. (2013). isomiRex: web-based identification of microRNAs, isomiR variations and differential expression using next-generation sequencing datasets. FEBS Lett. 587, 2629-2634. doi: 10.1016/j.febslet.2013.06.047 
Sablok, G., Pérez-quintero, Á. L., Hassan, M., Tatarinova, T. V., and López, C. (2011). Artificial microRNAs (amiRNAs) engineering-On how microRNAbased silencing methods have affected current plant silencing research. Biochem. Biophys. Res. Commun. 406, 315-319. doi: 10.1016/j.bbrc.2011. 02.045

Shao, C., Lu, G., Yu, L., Wu, Q., Chen, M., and Meng, Y. (2015). Identification of novel miRNA-target pairs in rice ('Oryza sativa') by a reversed approach Plant. OMICS 8, 62-68.

Sun, G. (2012). MicroRNAs and their diverse functions in plants. Plant Mol. Biol. 80, 17-36. doi: 10.1007/s11103-0119817-6

Tan, G. C., Chan, E., Molnar, A., Sarkar, R., Alexieva, D., Isa, I. M., et al. (2014). 5' isomiR variation is of functional and evolutionary importance. Nucleic Acids Res. 42, 9424-9435. doi: 10.1093/nar/ gku656

Wyman, S. K., Knouf, E. C., Parkin, R. K., Fritz, B. R., Lin, D. W., Dennis, L. M., et al. (2011). Post-transcriptional generation of miRNA variants by multiple nucleotidyl transferases contributes to miRNA transcriptome complexity. Genome Res. 21, 1450-1461 doi: 10.1101/gr.1180 59.110
Xie, F., Wang, Q., and Zhang, B. (2015). Global microRNA modification in cotton (Gossypium hirsutum L.). Plant Biotechnol. J. 13, 492-500. doi: 10.1111/pbi.12271

Zhang, B. (2015). MicroRNA: a new target for improving plant tolerance to abiotic stress. J. Exp. Bot. 66, 1749-1761. doi: 10.1093/jxb/erv013

Zhang, J., Zhang, S., Li, S., Han, S., Wu, T., Li, X., et al. (2013). A genome-wide survey of microRNA truncation and $3^{\prime}$ nucleotide addition events in larch (Larix leptolepis). Planta 237, 1047-1056. doi: 10.1007/s00425-012-1823-7

Conflict of Interest Statement: The authors declare that the research was conducted in the absence of any commercial or financial relationships that could be construed as a potential conflict of interest.

Copyright (c) 2015 Sablok, Srivastva, Suprasanna, Baev and Ralph. This is an openaccess article distributed under the terms of the Creative Commons Attribution License (CC BY). The use, distribution or reproduction in other forums is permitted, provided the original author(s) or licensor are credited and that the original publication in this journal is cited, in accordance with accepted academic practice. No use, distribution or reproduction is permitted which does not comply with these terms. 